\title{
The Web of Things
}

\author{
Matthew N. O. Sadiku, Omobayode I. Fagbohungbe, and Sarhan M. Musa \\ Roy G. Perry College of Engineering \\ Prairie View A\&M University \\ Prairie View, TX, USA
}

\begin{abstract}
The Web of Things may be regarded as an extension the Internet of Things that leverage Web-based standards and protocols to access and control real-world objects. While the Internet of Things (IoT) empowers communication between heterogeneous devices at the network layer, the web of things (WoT) enables interoperability at the application layer.

This paper provides a brief introduction to the web of things.
\end{abstract}

Key Words: Web of things, Social web of things.

\section{INTRODUCTION}

The web of things (WoT) describes approaches that allow real-world, physical objects to be part of the World Wide Web. Things can include consumer electronics, machines, RFID, barcodes, robots, sensors, actuators, cameras, mobile phones, smart meters, smart cars, traffic lights, smart home appliances, connected watches, etc. These objects could be fixed or mobile. The WoT provides an application layer that simplifies the applications of Internet of Things (IoT) [1,2]. Thus, WoT has emerged as the application platform for IoT. Things, people, and places congregate as friends on the Web based social networking platform.

The three basic steps to create web of things are:

1. Connecting things to the internet (IPv4 or IPv6); each object is identified with a specific IP address.

2. Embedded web servers; Smart things are connected to the Internet by embedding Web servers directly onboard.

3. Make devices part of web; more and more real world devices will become IP enabled and have embedded HTTP servers. Objects are integrated to the Web by reusing Web patterns such as REST (the Representational State Transfer). A typical architecture for WoT is shown in Figure 1 [4].

Web protocols (such as HTTP) and standards are a critical factor in the successful implementation of the WoT. HTTP, the Web's basic building block, enabled both page and file delivery through the server. The key idea is to represent resources on these devices as URIs and use HTTP verbs as the uniform interface to manipulate them [5].

\section{APPLICATIONS}

The web of things empowers future applications with new sources of data and innovative services with the ability to connect with the real-world and to control real things. The WoT is relevant for a wide variety of applications ranging from smart cities, agriculture, energy grids, and wildlife monitoring.

The Web of Things has been applied in smart homes to monitor and control the energy consumption of household appliances. It addresses the problem of heterogeneous home devices using Web technologies. New devices such as smart TVs, alarm clocks, and fridges make our houses more energy efficient and our lives more enjoyable. 
Information Retrieval (IR) is one form of open WoT-based applications, and one that is useful for smart cities. Conventional IR system performs two major functions- indexing and querying. The advances in real-time search constitute a basis for an IR system for the WoT [6].

\section{CHALLENGES}

Security concepts, such as authorization and ownership management, will be essential requirement for the large-scale WoT deployment. The WoT will depend on solutions to advanced authorization problems. Most physical things represented in the WoT will be proprietary items that legal entities will own [7].

WoT webservers can be mobile. Mobile IP and dynamic DNS are two potential ways to handle mobility. The web protocols have the biggest challenge of reducing the web latency that may significantly affect the real-time performance of WoT systems.

The heterogeneity at the devices (in terms of data communication methods and capabilities) seriously challenges the popularity of WoT.

\section{CONCLUSION}

The web of things can be regarded as a part of the Internet of things. WoT enables Devices to speak the same language, communicate, and interoperate freely on the Web.

The Web is continuously evolving and has already become the major medium communication in today's Internet. The goal of the web of things is to bring interoperability at the application layer, on top of the Internet of things.

Several implementations of WoT have been made [8,9]. The World Wide Web Consortium (W3C) is a member funded international organization that has the mission of leading the web to its full potential and developing initial standards. World $\mathrm{m}$

\section{REFERENCES}

[1] "Web of Things," Wikipedia, the free encyclopedia https://en.wikipedia.org/wiki/Web_of_Things

[2] M.N.O. Sadiku, and S.M. Musa and S. R. Nelatury, "Internet of things: an introduction," International Journal of Engineering Research and Advanced Technology, vol. 2, no.3, March 2016, pp. 39-43.

[3] V. K. Kollu, M. Manchikanti, and M. Srilatha, "The Web of Things," International Journal of Advanced Trends in Computer Science and Engineering, vol. 3, no.1, 2014, pp. 581- 584.

[4] D. Zeng, S. Guo, and Z. Cheng, "The Web of Things: A Survey (invited paper),"

Journal of Communications, vol. 6, no. 6, September 2011, pp. 424-438.

[5] V. Gupta, R. Goldman, and P. Udupi, "A Network Architecture for the Web of Things," Proceedings of the Second International Workshop on Web of Things, June 2011.

[6] C. Manta-Caro and J. M. Fern'andez-Luna, "Information retrieval as-a-service for the web of things: a survey and a proposal of IRaaS architecture," Proceedings of IEEE 4th International Conference on Future Internet of Things and Cloud, 2014, pp. 325333.

[7] D. Raggett, “The web of things: challenges and opportunities,” Computer, May 2015, pp. 26-32.

[8] B. Traversat et al., "Project JXTA-C: Enabling a web of things," Proceedings of the 36th Hawaii International Conference on System Sciences (HICSS’03), 2002.

[9] M. Blackstock and R. Lea, "WoTKit: A Lightweight toolkit for the Web of Things," Proceedings of the Third International Workshop on the Web of Things, June 2012.

\footnotetext{
ABOUT THE AUTHORS

Matthew N.O. Sadiku is a professor emeritus in the Department of Electrical and Computer Engineering at Prairie View A\&M University, Prairie View, Texas. He is the author of several books and papers. His areas of research interests include computational electromagnetics and computer networks. He is a fellow of IEEE.
}

Omobayode I. Fagbohungbe is a doctoral student at Prairie View A\&M University, Prairie View Texas. He holds a masters of science degree in control engineering from the University of Manchester, UK and a bachelor degree in electrical and electronics 
engineering from Obafemi Awolowo University, Nigeria. He is a graduate member of the IEEE. His current research interests are in data science, machine learning, and deep learning.

Sarhan M. Musa is a professor in the Department of Electrical and Computer Engineering at Prairie View A\&M University, Texas. He has been the director of Prairie View Networking Academy, Texas, since 2004. He is an LTD Sprint and Boeing Welliver Fellow. His research interests include computer networks and computational electromagnetics.

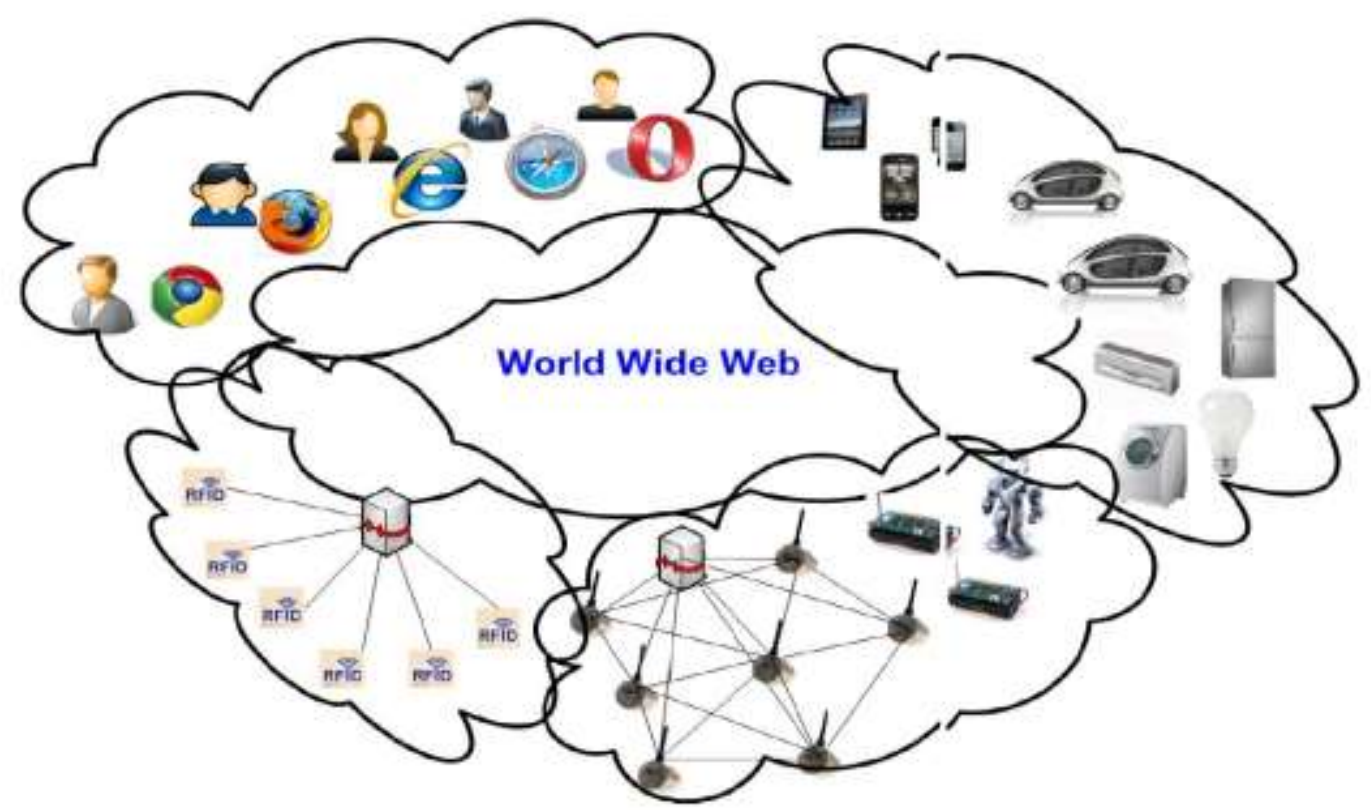

Figure 1 A typical architecture for web of things [4]. 